\title{
Occurrence bilateral bronchospasm consequently after unilateral bronchospasm
}

\author{
Yun Hee Kim, Woon Young Kim, Joong Il Kim, Doo Jae Min, and Yoon Sook Lee \\ Department of Anesthesiology and Pain Medicine, Korea University Ansan Hospital, Ansan, Korea
}

Patients, especially pediatric patients, with a recent history of an upper respiratory tract infection (URI) are at a significantly increased risk for the development of intraoperative airway complications due to airway hyperreactivity. For these patients, the incidence for bronchospasm can be 10 times the incidence for those patients who did not have recent URI [1]. We have managed a case of bilateral bronchospasm after unilateral bronchospasm that was not reported until now.

A 5-year-old, $18.65 \mathrm{~kg}, 87 \mathrm{~cm}$ female patient was scheduled for Hotz's operation for an entropion. During preoperative evaluation, her parents did report a history of URI symptoms 2 weeks prior to the visit. However, child appeared healthy without significant physical exam findings besides clear rhinorrhea. All of the preoperative laboratory studies were within normal ranges. Thus, the operation was scheduled without delay.

After the induction of anesthesia with thiopental $5 \mathrm{mg} / \mathrm{kg}$, rocuronium $0.6 \mathrm{mg} / \mathrm{kg}$ was administered for endotracheal intubation. A $5 \mathrm{~mm}$ cuffed endotracheal tube (ETT) was placed $15 \mathrm{~cm}$ away from the incisor. Upon ascultation, the left respiratory sounds were normal, but the right respiratory sounds were markedly decreased. A left endobronchial intubation was suspected, and the ETT was withdrawn by $1 \mathrm{~cm}$. The accuracy of tube positioning was re-evaluated by auscultation several times.

General anesthesia was maintained using 50\% oxygennitrous oxide mixture and sevoflurane (1.5-3.0 vol\%) according to blood pressure and heart rate under volume controlled ventilation. The peak inspiratory pressure (PIP) was $14 \mathrm{mmHg}$, and the end tidal carbon dioxide concentrations $\left(\mathrm{ETCO}_{2}\right)$ were between 30 and $35 \mathrm{mmHg}$. The vital signs remained stable. At the end of surgery, sevoflurane and nitrous oxide administration was discontinued. Within $3 \mathrm{~min}$ of this, the $\mathrm{SpO}_{2}$ decreased to 90\% with a high PIP (28 $\mathrm{mmHg})$. On auscultation, the left respiratory sounds were markedly decreased with a slight wheeze. Under the suspicion of a blocked tube, ETT was gently suctioned, but no secretion or blood was aspirated. Subsequently, an inadvertent right bronchus intubation was suspected from head movements during the operation, and the tube was withdrawn in stepwise increments while lung fields were auscultated. During this process, the tube was unintentionally extubated out of the trachea, and the patient was reintubated after mask ventilation using 100\% oxygen and sevoflurane (3.0-5.0 vol\%). The tracheal tube position was confirmed by fiberoptic bronchoscopy. Approximately 10 minutes after intravenous administration of hydrocortisone $50 \mathrm{mg}$, the $\mathrm{SpO}_{2}$ improved to $99 \%$, and the PIP decreased to $15 \mathrm{mmHg}$ with clear breath sound in both lung fields. Sevoflurane was discontinued again. After an additional 10 minutes, the $\mathrm{SpO}_{2}$ gradually dropped to under $70 \%$ with marked decreases in tidal volume. Again, wheezing was heard in both lung fields. Because of this, the patient was manually ventilated with $100 \% \mathrm{O}_{2}$ and sevoflurane (3.0 vol\%), with the PIP measurements over $35 \mathrm{cmH}_{2} \mathrm{O}$ at this point. Two puffs of ventolin (albuterol) from a metered dose inhaler were discharged down the ETT, and intravenous atropine $0.1 \mathrm{mg}$ was administered. With the bronchodilation therapy, $\mathrm{SpO}_{2}$ returned to $99 \%$, while PIP gradually decreased under $20 \mathrm{mmHg}$. The wheezing disappeared and the tidal volume increased near to the initial levels. A chest X-ray did not reveal any specific pulmonary changes with the end of ETT positioned $1.0 \mathrm{~cm}$ above

Corresponding author: Woon Young Kim, M.D., Ph.D., Department of Anesthesiology and Pain Medicine, Korea University Ansan Hospital, 516, Gojan 1-dong, Danwon-gu, Ansan 425-707, Korea. Tel: 82-31-412-5291, Fax: 82-31-412-5294, E-mail: ckssis@korea.ac.kr (c) This is an open-access article distributed under the terms of the Creative Commons Attribution Non-Commercial License (http:// creativecommons.org/licenses/by-nc/3.0/), which permits unrestricted non-commercial use, distribution, and reproduction in any medium, provided the original work is properly cited. 
the carina. After the bronchodilator treatment, extubation and other postoperative courses were uneventful.

Unilateral bronchospasms are clinically similar to those of bronchial obstruction or a unilateral mainstem bronchus intubation. Because it is a relatively rare condition, a unilateral bronchospasm is easy to misdiagnose, as was the experience in this case. Therefore, unilateral bronchospasms should be seriously considered in the differential diagnosis of a patient with decreased unilateral lung sounds with or without wheeze.

Bronchospasm is defined as a narrowing of the respiratory tract, which is caused by an abnormal contraction of the smooth bronchial muscle. Signs of bronchospasm include increased PIP, prolonged respiration, audible wheezing, rising $\mathrm{ETCO}_{2}$, and a reduction in tidal volumes. It is known that bronchospasm occurred due to airway irritation in patients who have predispositions towards chemical, mechanical, or neurogenic stimulation [2].

Unilateral bronchospasm is reported with less frequency then for bilateral bronchospasm. Shantha [3] reviewed the possible mechanism of unilateral bronchospasm, which may be an imbalance between sympathetic and parasympathetic nervous tones. In the anesthesia literature, unilateral bronchospasm are reported in the context of interpleural analgesia, subclavian vein puncture, microcatheter manipulation, inadvertent topical lidocaine injection into the bronchus, and inadequately anesthetic depth [3-5]. In this case, the left unilateral bronchospasm was believed to be caused by airway hypersensitivity from a recent URI during the light anesthesia in the emergence period. Additionally, inadvertent left-side endobronchial intubation could have contributed as a mechanical airway stimulus.

In the case presented, the early unilateral bronchospasm was not detected because occurrence of unilateral bronchospasm is extremely unlikely when compared to the initial suspicion of blocked ETT by thick secretions and mucous plug. The secondary suspicion was an inadvertent mainstem bronchus intubation. In children, ETT can easily be misplaced with neck flexion or extension, which may result in inadvertent mainstem bronchus intubation or extubation. In many cases, endobronchial intubation is easily managed by withdrawal of the tip ETT to above the carina. In our case, we tried to correct the depth of ETT placement by withdrawing the ETT but ended up unintentionally extubating the patient. Subsequent to this, the ETT was reintubated with high concentration of sevoflurane (3.0-5.0 vol\%). During the re-emergence, bilateral bronchospasm occurred in the absence of anaphylactic symptoms such as hives, flushing skin rash and severe hypotension. We aggressively treated bronchospasm using endotracheal atomized albuterol spray and intravenous atropine with high concentrations of sevoflurane.

Intraoperative bronchospasm is a potentially life-threatening complication of anesthesia, especially in infants and children. We emphasize that prevention is the best therapy. Awareness of various precipitating factors of bronchospasm is important because adverse perioperative respiratory events can be anticipated and are easy to recognizable and treat. In addition, we must suspect an unilateral bronchospasm when lung sounds are markedly decreased in an unilateral lung field with or without wheeze.

\section{References}

1. Lopez-Gil M, Brimacombe J, Alvarez M. Safety and efficacy of the laryngeal mask airway. A prospective survey of 1400 children. Anaesthesia 1996; 51: 969-72.

2. Canning BJ. Reflex regulation of airway smooth muscle tone. J Applied Physiol (1985) 2006; 101: 971-85.

3. Shantha TR. Unilateral bronchospasm after interpleural analgesia. Anesth Analg 1992; 74: 291-3.

4. Reddy K, Prabhakar H, Yadav N, Singh GP, Ali Z. Unilateral bronchospasm during microcatheter manipulation in an interventional neuroradiology suite. J Anesth 2010; 24: 313-4.

5. Farmery AD. Severe unilateral bronchospasm mimicking inadvertent endobronchial intubation: a complication of the use of a topical lidocaine Laryngojet injector. Br J Anaesth 2000; 85: 917-9. 